\title{
Anabases
}

ANABASES Traditions et réceptions de l'Antiquité

4 | 2006

Varia

\section{Quelques réflexions sur l'analyse historiographique et épistémologique du DAGR}

\author{
Monique Dondin-Payre
}

\section{OpenEdition}

Journals

Édition électronique

URL : http://journals.openedition.org/anabases/2970

DOI : 10.4000/anabases. 2970

ISSN : 2256-9421

Éditeur

E.R.A.S.M.E.

Édition imprimée

Date de publication : 1 octobre 2006

Pagination : 169-172

ISSN : 1774-4296

\section{Référence électronique}

Monique Dondin-Payre, «Quelques réflexions sur l'analyse historiographique et épistémologique du DAGR », Anabases [En ligne], 4 | 2006, mis en ligne le 01 janvier 2012, consulté le 20 octobre 2019. URL http://journals.openedition.org/anabases/2970; DOI : 10.4000/anabases.2970 


\section{Quelques réflexions sur l'analyse historiographique et épistémologique du $D A G R$}

MoniQue Dondin-PAyre

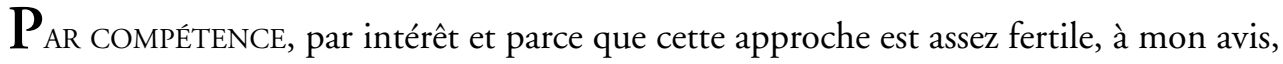
pour justifier un programme, je me borne ici à quelques réflexions sur l'analyse historiographique et épistémologique du $D A G R$ en lui-même. Il ne s'agit pas de confronter le Dictionnaire à la science actuelle - ce qui a été l'objet de la seconde partie de la journée d'étude ERASME et constitue le second volet de cette publication -, mais d'en chercher la logique interne, de repérer les éventuels infléchissements au cours de la réalisation, et leurs causes.

\section{Le contexte}

À partir de la contribution très précise de Catherine Valenti ${ }^{1}$, on pourrait approfondir l'impact du contexte :

- le positivisme (le mot n'a pas été prononcé, mais il mérite de l'être)

Il imprègne la démarche de Daremberg et se reflète dans le $D A G R$. En témoigne le goût de Daremberg pour les manuscrits, pour la précision scientifique médicale, pour la valeur de la preuve. Il a tenu à faire passer la médecine d'une science livresque à une science "de laboratoire ", fondée sur des expérimentations concrètes. S'il se situe donc dans une revendication d'érudition que l'on a pu qualifier de "tatillonne ", il vit aussi dans un monde où c'est une exigence intellectuelle et morale que de partager cette érudition, dans des conditions telles qu'elle ne soit pas réservée à une élite. 
De là découle directement la question de l'introduction des illustrations dans le Dictionnaire: c'est une grande nouveauté, comme on l'a bien souligné ${ }^{2}$. Elle correspond aux critères positivistes, à une démarche probatoire, et aussi à la facilité de compréhension qu'apporte le fait de montrer. La démonstration du fait qu'il s'agit d'une innovation pédagogique est apportée par leur reprise dans ce qui s'appelait alors le Dictionnaire illustré latin-français de Gaffiot. Certes, l'opération est commerciale, comme il a été dit, puisque les dessins sont disponibles et libres de droits, mais il eût été encore plus économique de n'inclure aucune illustration, comme on le faisait jusque là ; et Félix Gaffiot était trop exigeant pour accepter des solutions au rabais. Si les dessins ont été inclus tels quels, c'est qu'ils répondaient à des exigences pédagogiques et intellectuelles ;

\section{- le souci de la diffusion de l'éducation}

Avait-on demandé ce dictionnaire presque par hasard à Daremberg, ou plutôt parce qu'il en avait déjà fait d'autres, qui avaient connu de réels succès commerciaux ? Poser la question en ces termes, c'est, je crois, la prendre à l'envers et de manière superficielle. Certes, Daremberg avait fait preuve de son sens de la vulgarisation, mais il a été sollicité parce que la confection de dictionnaires correspondait à sa conception de la science et à son désir de la partager. Nous venons, à cet égard, d'évoquer son souci de diffuser la science médicale approfondie et expérimentale auprès de collègues moins aguerris.

On soulignera, dans cette perspective, deux aspects significatifs :

a) la position du DAGR par rapport à l'Encyclopédie de Diderot: je suis frappée de voir combien, sur certains points, les deux œuvres se ressemblent (le goût du concret, l'attention aux métiers, au quotidien, aux ustensiles, le tout mis à portée du public par les illustrations); un ou plusieurs des concepteurs ou rédacteurs se sont-ils explicitement référés à l'Encyclopédie, antérieure d'un siècle exactement ? Ceci pose la question des modèles adoptés par Daremberg et ses collaborateurs ;

b) le contexte contemporain : on marche vers l'instruction obligatoire pour tous les enfants ; l'éducation a pour objectif de former de meilleurs citoyens, plus à même de voter en connaissance de cause et d'apporter une contribution à la société. Sans être à la portée de tous, le DAGR n'est pas un ouvrage abscons et il est même conçu pour, et utilisable par, les gens cultivés.

\section{Les illustrations}

Elles suscitent un certain nombre d'interrogations, parmi lesquelles les suivantes : - pourquoi l'origine des illustrations n'est-elle pas clairement indiquée par des légendes ? Peut-on, dès lors, envisager, dans la version en ligne, de retrouver et de préciser systématiquement la référence des documents 3 ?;

2 Cf. la contribution de F. Lissarague, p. 173-177.

3 Ibid. 
- les illustrations sont-elles toutes des versions gravées de documents originaux, comme on le faisait alors très couramment pour les publications savantes (voir les publications des travaux des commissions d'exploration) ? ou, par souci pédagogique, certains documents sont-ils combinés pour être plus en rapport avec le texte ? à moins que, à l'inverse, ils ne soient simplifiés?

Bref, comment l'image est-elle traitée sur le plan technique, pédagogique et scientifique dans le $D A G R$ ? Que pouvons-nous en faire aujourd'hui ?

\section{Les auteurs}

\section{a) la place de Daremberg}

Même s'il est mort tôt, il ne faut pas sous-estimer l'importance du premier concepteur. Il a pris une part limitée (ou nulle ? à vérifier) à la rédaction des notices. Mais c'est lui qui a fixé l'orientation de l'entreprise et qui a accumulé les documents et les informations ${ }^{4}$. D. Gourevitch a bien étudié la part de Daremberg dans la conception du Dictionnaire; elle la juge essentielle et, une fois l'impulsion donnée par lui, il me semble qu'on s'en est tenu à ses principes. Est-ce une fausse impression ? Peut-être dans les volumes de documents que sa femme a joints à sa bibliothèque quand elle l'a vendue et que D. Gourévitch dit non exploités, y a-t-il des informations complémentaires qui permettraient d'affiner cette appréciation ? C'est une piste qui mériterait d'être suivie.

On pourrait peut-être aussi tenter d'apprécier l'évolution qu'a probablement connue cette entreprise de longue haleine, en prenant dans le premier tome une notice générale et en scrutant les approches dans les notices apparentées au sein des volumes suivants : convergent-elles ou divergent-elles ? remarque-t-on des écarts, des infléchissements dans la manière de traiter les sujets ? Donc, travailler sur l'histoire interne du $D A G R$ et ses relations avec l'évolution de la science historique en général.

\section{b) les contributeurs}

Il serait intéressant de savoir exactement comment les trois concepteurs, Daremberg, Saglio et Pottier se sont connus et pourquoi ils ont collaboré à l'entreprise du DAGR. Estce l'éditeur qui a fait le lien entre eux ? Se connaissaient-ils par ailleurs? On entre là dans le domaine de la sociabilité savante qui est important pour comprendre le fonctionnement de telles initiatives collectives. Il est certain que Saglio et Pottier se recom-

4 D. Gourevitch, Charles Victor Daremberg (1817-1872) et une histoire positiviste de la médecine, BIUM : http ://www.bium.univ-paris5.fr/histmed/medica/daremberg.htm. 
mandaient par leurs domaines de compétence, dans le cadre desquels ils ont amplement travaillé, mais d'autres auraient pu leur être préférés.

Une fois pareille entreprise lancée, on recrute des contributeurs soit en fonction des thèmes prévus, soit en fonction des compétences rencontrées par hasard, sans doute les deux à la fois : a-t-on inséré des notices parce qu'on connaissait quelqu'un qui avait des aptitudes pour les traiter ? Ou a-t-on respecté strictement le schéma initial, s’il y en avait un ? La correspondance entre Pottier et Cumont apporte un éclairage significatif sur ces tâtonnements 5 . Il n'est pas très compliqué, en effet, de prévoir des entrées un peu biaisées pour qu'elles correspondent à une personne, plutôt que de recruter un auteur parce qu'il connaît un sujet prédéterminé. Il est probable que le glossaire récapitulatif donne des idées sur ce point.

On s'interrogera aussi sur les absents, donc sur la politique de recrutement de la part du trio des concepteurs. Qui, parmi les savants connus, n'a pas été recruté ? et pourquoi ? quel est le profil du collaborateur idéal ? Il serait utile de dresser un panorama des figures de la science historique de l'époque pour déterminer qui a été retenu et qui ne l'a pas été. Les correspondances devraient pouvoir nous renseigner sur l'entourage scientifique, proche et lointain de Daremberg, Saglio et Pottier. Qu'en a-t-on conservé ? et où ?

Parmi les auteurs impliqués dans le projet, les moins connus, les plus modestes et obscurs sont également intéressants. D’où sortent-ils ? comment ont-ils été contactés ? pourquoi ont-ils été retenus ? Quelle est l'implication des chercheurs confirmés et des jeunes recrues? Y a-t-il des formes de sous-traitement des entrées de la part d'autorités reconnues?

Il serait également intéressant de s'interroger sur la place de la collaboration au $D A G R$ dans les carrières : j'ai eu l'impression que, pour ceux qui sont devenus " célèbres ", leur contribution se place en début de carrière. La participation au Dictionnaire serait donc non pas une consécration, mais un moyen de rendre service ou de se faire connaître, une rampe de lancement, dans le meilleur des cas. Alors, au fil du temps peut-être (pas dès le début), la participation au DAGR pourrait être une stratégie de carrière en même temps que (ou uniquement ?) une contribution à une ouvre nationale, à situer dans un contexte francophone militant.

Il me semble, enfin, que la place de l'Académie des Inscriptions et Belles Lettres n'a pas été évaluée à sa juste valeur ; c'est le carrefour de la science de l'Antiquité à l'époque, et les collaborateurs qui sont entrés à l'Académie après avoir travaillé au DAGR ont toute chance d'avoir été recrutés par ce canal, parce qu'ils assistaient aux séances, aux cours des académiciens dans les institutions, parce qu'ils leur servaient de secrétaires, de documentalistes...

MONIQUe DONDIN-PAYRE

CNRS - UMR 8585

dondin_payre@club-internet.fr 